\title{
JURISPRUDENCIA CONSTITUCIONAL EN MATERIA DE PROTECCIÓN \\ DEL MEDIO AMBIENTE \\ (PRIMER SEMESTRE 2021)
}

\author{
CLARA EsteVE JoRDÀ \\ Investigadora Predoctoral Contratada Martí i Franquès \\ Universitat Rovira i Virgili \\ clara.esteve@urv.cat
}

SUMARIO: 1. De la regulación autonómica de las actividades cinegéticas. 2. Más sobre la constitucionalidad de la regulación autonómica de caza: mismo objeto, distintos procedimientos. 3. De la regulación autonómica de la acción pública ambiental por vía jurisdiccional.

\section{De la regulación autonómica de las actividades cinegéticas}

La Sentencia 148/2020, de 22 de octubre de 2021 (BOE núm. 305, de 20 de noviembre de 2020) resuelve el recurso de inconstitucionalidad interpuesto por el Defensor del Pueblo en relación con el artículo único, apartados 1, 3, 5 y 6, y la disposición transitoria de la Ley 9/2019, de 28 de marzo, de modificación de la Ley 4/1996, de caza en la comunidad Autónoma de Castilla y León. El apartado 1 de esta nueva redacción modifica el art. 7 de dicha ley (especies cinegéticas cazables) mientras que el apartado 3 modifica su art. 42 (limitación de los periodos hábiles de caza). El apartado 5 añade un anexo I, donde se incluye el listado de especies cinegéticas, mientras que el apartado 6 incorpora un anexo II, en el que se relacionan los periodos y días hábiles para el ejercicio de la caza. Por su parte, la disposición transitoria determina que "En tanto se apruebe el plan general de caza de Castilla y León, la caza se practicará conforme a las disposiciones de esta ley".

La impugnación del Defensor del Pueblo se fundamenta en dos motivos distintos. En primer lugar, considera que los preceptos impugnados vulneran la legislación 
básica estatal en materia de medio ambiente. Concretamente, los arts. 54.1 y 5 ; 61.1 y 65 de la Ley 42/2007, de 13 de diciembre, de patrimonio natural y de la biodiversidad (LPNB, en adelante). Según el Defensor, los preceptos recurridos fueron aprobados sin los estudios necesarios para garantizar la adecuada protección de las especies silvestres, de modo que infringían los arts. 49 y 149.1.23 CE. El segundo argumento esgrimido es que las prescripciones impugnadas se encuentran en una ley singular, aprobada con la única finalidad de eludir el cumplimiento y dejar sin efectos determinadas resoluciones judiciales, de forma que vulneraban los arts. 9.3 y 24 y 24.1 CE. Formularon alegaciones ante este recurso la representación tanto de la Junta como de las Cortes de Castilla y León, afirmando la constitucionalidad de la Ley impugnada y defendiendo la importancia de la actividad cinegética —esto es, la caza - en esta comunidad, como herramienta de gestión y de interés social.

Entremos a analizar el primero de los argumentos planteados por el Defensor del Pueblo, de índole competencial. El Tribunal arguye que la regulación controvertida fue dictada conforme a lo dispuesto en el art. 70.1.17 del Estatuto de Autonomía de Castilla y León, que define la competencia exclusiva en materia de caza y explotaciones cinegéticas. Aunque el art. 149.1.23 CE reserva al Estado la legislación básica en materia de medio ambiente, es reiterada la doctrina que ha señalado que ello no es óbice para el diseño y la ejecución de legislación autonómica en materia de caza y de protección de los ecosistemas vinculados directamente a esta actividad (SSTC 14/1998, FJ 2; 69/2013, FJ 3 y 79/2019, FJ 5).

EI TC confirma el carácter básico, material y formal de la LPNB estatal, que actúa de parámetro de contraste de la norma autonómica y tiene capacidad para incidir transversalmente en el ejercicio de las competencias autonómicas en materia de caza. Para ello, el garante de la Constitución trae a colación la STC 69/2013, FJ 1 y 6 , argumentando que el criterio decisivo para calificar una norma de protección del medio ambiente como básica es su propia finalidad tuitiva. En este caso, la LPNB contiene una serie de prescripciones generales y comunes de carácter mínimo que inciden en el ejercicio de la actividad cinegética, orientada a la conservación de la biodiversidad de las especies silvestres. $Y$ es que las actividades de caza no solo deben desarrollarse conforme a los requisitos 
establecidos por la legislación autonómica para su ejercicio, sino también a los de protección del medio ambiente.

Alega el Defensor del Pueblo en su escrito de demanda que, según la exposición de motivos de la Ley autonómica 9/2019, la declaración de determinadas especies como cinegéticas depende de dos requisitos: por un lado, que no se encuentren en ninguno de los supuestos de protección conforme a la normativa comunitaria, estatal y autonómica; y por otro que, conforme a su población, distribución y reproductividad puedan soportar una extracción ordenada de ejemplares. No obstante, el recurrente solo cuestiona el segundo de los requisitos. Argumenta que la modificación no ha sido aprobada con los exigibles informes y estudios científicos y técnicos que respalden su adecuación al deber básico de conservación de las especies afectadas, y que acrediten que este no se verá disminuido por la práctica de la caza. Sin embargo, no menciona cuáles deberían ser estos informes ni alude a ninguna norma específica autonómica que los contemple como exigibles, como tampoco razona por qué tales disposiciones atentarían contra el deber general de protección de la fauna silvestre. Para apreciar si incurre la sugerida vulneración del procedimiento legislativo en la aprobación de la ley autonómica, el Tribunal recuerda que:

"En primer lugar ha de determinarse (...) la norma reguladora del procedimiento de elaboración de las leyes que ha resultado infringida y, en segundo término, una vez localizada tal infracción, ha de evaluarse después la magnitud del defecto de procedimiento detectado, que solo adquiere dimensión constitucional si es susceptible de afectar no de cualquier manera, sino de forma sustancial, el proceso de formación de la voluntad de una cámara, incidiendo, en consecuencia, al ejercicio de la función representativa inherente al estatuto del parlamentario (...). De este modo, una vez detectada la fuente normativa aplicable, la ausencia de un determinado antecedente solo tendrá transcendencia si se hubiere privado a las cámaras de un elemento de juicio necesario para su decisión. (...) Es por tanto imprescindible que el defecto denunciado tenga cobertura normativa en el propio ordenamiento autonómico, de suerte que exista una violación específica del bloque de constitucionalidad." (STC 70/2018, de 21 de junio, FJ 4 b). 
El Tribunal Constitucional afirma que el texto de la proposición sí cumple con las formalidades legales. La ley se presenta de forma articulada, acompañada de unos antecedentes y una exposición de motivos detallando las razones que sustentan la modificación normativa, siguiendo el procedimiento previsto de conformidad con lo dispuesto en el bloque de constitucionalidad aplicable (el art. 121 del Reglamento de Castilla y León). Descarta pues, el Tribunal, la violación del procedimiento legislativo seguido en la aprobación de la norma, al no haberse aportado carga argumental suficiente y haberse formulado la controversia "con carácter hipotético y preventivo". Por este motivo rechaza la inconstitucionalidad de la impugnación formulada por el Defensor del Pueblo.

Profundicemos ahora en el segundo de los motivos aducidos por el Defensor del Pueblo: la arbitrariedad. Manifiesta éste que los preceptos impugnados vulneran el art. 9.3 CE porque se trata de una ley singular, en base a tres motivos. Primero, que los anexos y la disposición transitoria son "parcialmente autoaplicativos", al no requerir la emisión de acto administrativo de aplicación. En segundo lugar, que se trata de una ley de supuesto de hecho concreto. $Y$, finalmente, que no se justifica la excepcional relevancia de la modificación por ley de una materia antes regulada reglamentariamente, siendo la reforma desproporcionada. Se afirma, asimismo, la vulneración del derecho a la tutela judicial efectiva ex art. 24.1 CE, al impedir el acceso a la justicia de los titulares de derechos e intereses legítimos y privar de efectividad a las resoluciones judiciales relacionadas con la materia objeto de la norma cuestionada. Cabe tener en cuenta que el reglamento que antes regulaba la actividad cinegética se encontraba impugnado en vía judicial durante la aprobación de la nueva ley autonómica.

El alto tribunal descarta que la norma cuestionada sea una ley singular, ya que regula con carácter general y abstracto la actividad cinegética de Castilla León, no agotándose su eficacia con la aplicación a un caso concreto. Además, la norma va dirigida a una pluralidad de sujetos indeterminada; todos aquellos que confluyen en el legítimo ejercicio de la actividad cinegética, y su vocación es de permanencia, susceptible de múltiples aplicaciones. El Tribunal descarta también la infracción de los arts. 9.3 y $24.1 \mathrm{CE}$, dado que no aprecia desproporción en la decisión del legislador autonómico de regular la actividad cinegética. Al fin y al cabo, existe libertad del legislador para elegir el nivel de 
densidad normativa con que regula una determinada materia ${ }^{1}$. $\mathrm{Y}$ concluye el Tribunal, para desestimar el recurso de inconstitucionalidad, que:

“(...) no corresponde a este tribunal interferirse en el margen de apreciación que corresponde al legislador democrático, ni examinar la oportunidad de la medida legal para decidir si es la más adecuada o la mejor de las posibles, sino examinar si la decisión adoptada es plenamente irrazonable o carente de justificación" (SSTC 156/2014, FJ 6 y 110/2015, FJ 5).

En relación con esta libertad del legislador autonómico cabe destacar que, en Castilla y León, más del $88 \%$ de su superficie son terrenos cinegéticos, la mayor parte constituidos bajo la figura de cotos privados de caza. Se defiende en la exposición de motivos de la ley impugnada que la actividad cinegética es la herramienta más eficaz para limitar los daños ocasionados por las poblaciones de ungulados silvestres, jabalíes y conejos, sobre los cultivos, la ganadería y los accidentes de tráfico. Pero también se defiende la ley por motivos económicos, ya que los ingresos obtenidos de la caza constituyen la principal fuente de riqueza de las comarcas más rurales y menos industrializadas, y por tanto, se evita la despoblación del medio rural en esta comunidad autónoma.

Al no mencionarse en el texto de la demanda del Defensor los supuestos informes técnicos omitidos, nunca resulta probado que la regulación autonómica permita efectivamente el ejercicio de la caza en términos compatibles con la conservación de la biodiversidad. Lo cierto es que no parece tan clara la finalidad de la ley autonómica de proteger a la fauna silvestre, como señala el Tribunal, o de salvaguardar el interés ecológico como manifestación del interés general y público para la preservación de la riqueza biológica, escasa y fácilmente extinguible. No se puede obviar que la norma destila también la voluntad del legislador castellanoleonés de salvaguardar los intereses económicos de una región que lidera el descenso de población en España desde hace años ${ }^{2}$. Si bien,

\footnotetext{
${ }^{1}$ El preámbulo de la ley castellanoleonesa de caza ya advertía, de hecho, de la necesidad de solucionar problemas como la superpoblación de especies silvestres en materia de seguridad vial, salud humana, seguridad alimentaria, daños en cultivos, importancia económica de la actividad cinegética en zonas rurales y sus efectos en términos de despoblación, así como la amenaza de extinción de algunas especies debido a la sobrepoblación de otras.

${ }^{2}$ Alba Camazón, "La fallida agenda contra la Castilla y León vacía: la Comunidad pierde en 10 años la población equivalente a la provincia de Palencia, Ávila o Segovia", 8 de noviembre de 2020 en ElDiario.es <https://www.eldiario.es/castilla-y-leon/sociedad/fallida-agenda-castilla-
} 
evidentemente, no compete al Tribunal Constitucional entrar a razonar qué tan justificables son estos intereses.

\section{Más sobre la constitucionalidad de la regulación autonómica de caza: mismo objeto, distintos procedimientos}

El mismo 22 de octubre en que se dictó la recién comentada sentencia 148/2020, el Tribunal Constitucional también resolvió sobre una cuestión de inconstitucionalidad planteada por la Sección Primera de la Sala de lo Contencioso-Administrativo del Tribunal Superior de Justicia de Castilla y León (en adelante, TSJCL). Sala que, conviene conocer, había dictado la suspensión cautelar del reglamento que antes regulaba la actividad cinegética en esta comunidad autónoma. En esta ocasión, la STC 149/2020 resuelve sobre la constitucionalidad del artículo único, la disposición transitoria y la disposición derogatoria de la ya citada Ley 9/2019, de 28 de marzo, de modificación de la Ley de caza de Castilla y León.

Entre las dudas de constitucionalidad de la Sala del TSJCL, salta de nuevo el planteamiento de que la norma autonómica cuestionada es una ley singular autoaplicativa. Se aduce que legisla de modo autosuficiente el ejercicio de la actividad cinegética, que antes regulaba un reglamento, y que fue dictada en atención a un supuesto de hecho concreto y singular. Esto podría impedir a los tribunales el ejercicio de su potestad de juzgar y ejecutar. Asimismo, señala la Sala del TSJCL que ella misma dictó una medida cautelar que imposibilitaba la práctica de la caza de Castilla y León, con lo cual la finalidad de la ley habría sido permitir la práctica de dicha actividad y privar de efecto la medida cautelar. Por ello, considera la Sala que esta norma infringe el principio de separación de poderes, al vulnerar el art. 117.3 CE en relación con el art. 24.1 CE, el derecho a la ejecución y al cumplimiento de las resoluciones judiciales (arts. 118 y 24.2 CE) y el principio de interdicción de la arbitrariedad consagrado en el art. 9.3 CE. La consideración se efectúa en general, respecto de todos los preceptos impugnados conjuntamente. Como la singularidad de la ley cuestionada ya fue 
resuelta en la sentencia precedente a esta, el Tribunal Constitucional utiliza exactamente los mismos argumentos para llegar a la conclusión de que no puede ser calificada como ley singular. En consecuencia, no entra a examinar la constitucionalidad de la ley castellanoleonesa de caza.

Sobre la cuestión de la vulneración de los arts. 117.3 CE (exclusividad de jueces en el ejercicio de la actividad jurisdiccional) y 24.1 CE (tutela judicial efectiva) y del principio de separación de poderes, la Sala alegaba que al otorgarse rango de ley a una materia antes regulada por reglamento, se impedía al poder judicial controlar la determinación de las especies cinegéticas cazables, los periodos y días hábiles para cazar y el resto de la regulación de la actividad cinegética. Tras citar varias sentencias, el Tribunal declara nuevamente que no existe reserva reglamentaria en el ordenamiento jurídico español, y desestima la vulneración constitucional de los preceptos cuestionados, ya que ningún principio constitucional de los señalados prohíbe al legislador dictar una regulación general que pueda tener incidencia en los procesos en curso (como es el caso). La pretendida vulneración del derecho a la ejecución y cumplimiento de las resoluciones judiciales (arts. 118 y $24.2 \mathrm{CE}$ ), se sostenía en que la ley autonómica impedía la ejecución y cumplimiento de la suspensión cautelar de la actividad cinegética acordada por la propia Sala. El Tribunal Constitucional, no obstante, no aprecia inconstitucionalidad, ya que la resolución judicial no impedía que el legislador pudiera dictar una ley que regulara y permitiera el ejercicio de la caza. EI TC insiste en el carácter general y abstracto de la norma autonómica para declarar que el hecho de que pudiera determinar la pérdida de objeto de las medidas cautelares no la convertía en una ley singular que pretendiera evitar el cumplimiento de una resolución judicial.

Respecto a la posible vulneración del principio de interdicción de la arbitrariedad de los poderes públicos, no constata el alto tribunal falta de explicación racional sobre el objeto regulado, rechazando así la inconstitucionalidad de la infracción del art. 9.3 CE. EI TC reconoce que la actividad cinegética incide en muchos ámbitos (medio ambiente, salud pública, agricultura...) pero recalca la especial importancia socioeconómica como justificación de su regulación por parte del legislador. Y para ello se remite, como en la anterior sentencia, a la exposición de motivos de la ley castellanoleonesa. Concluye que establece un régimen 
complementario de protección de las especies silvestres para garantizar que el ejercicio de la caza no compromete su estado de conservación. Por todo ello, y siguiendo el camino argumental abierto en la primera sentencia dictada el 22 de octubre, el Tribunal Constitucional desestima la cuestión de inconstitucionalidad planteada por la Sala de lo Contencioso-Administrativo del TSJCL.

\section{De la regulación autonómica de la acción pública ambiental por vía jurisdiccional}

Como es sabido, la acción pública —también denominada popular-implica el reconocimiento de la legitimación activa para la defensa de la legalidad sin necesidad de invocar la lesión de un derecho ni un interés subjetivo. De este modo, cualquier sujeto individual o colectivo puede impugnar, ya sea ante la Administración o bien ante los jueces y tribunales, la actividad administrativa que considere contraria a derecho. La acción pública se erige, pues, como una excepción a la exigencia de la concurrencia de un interés directo o legítimo para recurrir un acto administrativo.

En la presente ocasión, la Sentencia 15/2021, de 28 de enero de 2021 (BOE núm. 46, de 23 de febrero de 2021) abre un melón complejo y despierta dudas sobre la legislación autonómica reguladora de la acción pública por vía jurisdiccional. Al menos, en materia de medio ambiente. Dicha sentencia enjuicia una cuestión de inconstitucionalidad planteada por la Sección Segunda de la Sala de lo Contencioso-Administrativo del Tribunal Superior de Justicia del País Vasco. El precepto de dudosa constitucionalidad es el inciso final del art. 3.4 de la Ley del Parlamento Vasco 3/1998, de 27 de febrero, general de protección del medio ambiente del País Vasco. Dicho artículo disponía que: "Será pública la acción para exigir el cumplimiento de lo previsto en esta ley, tanto en vía administrativa como jurisdiccional'.

La duda, de carácter competencial, plantea que el inciso - "como jurisdiccional" - podría vulnerar el art. 149.1.6 CE, que recoge la competencia exclusiva del Estado sobre legislación procesal. Al disponer que es pública la acción para exigir en vía jurisdiccional el cumplimiento de la Ley ambiental vasca, el legislador autonómico podría estar excediendo el ámbito de sus competencias. 
La postura planteada por el órgano jurisdiccional vasco es compartida por la Fiscal General del Estado, el abogado del Estado, la Diputación Foral de Gipuzkoa y el Consorcio de Residuos de Gipuzkoa. Por contra, el Gobierno y el Parlamento Vasco, así como la asociación Gurasos Elkartea, consideran que el precepto impugnado es una norma adicional de protección en materia medioambiental (art. 149.1.23 CE).

La principal duda que debe despejar el Tribunal Constitucional es si la acción del art. 3.4 de la ley vasca debe encuadrarse como legislación procesal o bien sectorial (de protección del medio ambiente). El Tribunal determina que la regla general del art. 149.1.6 CE prevalece por sobre de la regla competencial sectorial de medio ambiente. De no ser así, se vaciaría de contenido la competencia sobre legislación procesal. El TC se sirve de abundante jurisprudencia para reforzar que:

“(...) la legislación procesal es una «competencia general» del Estado [SSTC 80/2018, de 5 de julio, FJ 5 a); 13/2019, de 31 de enero, FJ 2 b), y 65/2020, de 18 de junio, FJ 16 B)] que responde a la necesidad de salvaguardar la uniformidad de los instrumentos jurisdiccionales [SSTC 71/1982, de 30 de noviembre, FJ 20; 83/1986, de 26 de junio, FJ 2, y 173/1998, de 23 de julio, FJ 16 c)]; y una competencia autonómica «de orden limitado» circunscrita a «las necesarias especialidades que en este orden se deriven de las particularidades del derecho sustantivo de las comunidades autónomas». [STC 80/2018, FJ 5 a)]."

Enmarcada pues, la duda constitucional como competencia en materia procesal y no ambiental, el Tribunal declara que, aunque el art. 149.1.6 CE no establece reserva absoluta en favor del Estado de legislación procesal, las singularidades procesales que se permiten legislar a las comunidades autónomas han de limitarse solo a aquellas que lo requieran por estar conectadas directamente con las particularidades del derecho sustantivo autonómico. Para determinar si una norma procesal autonómica constituye o no una necesaria especialidad procesal, deben efectuarse tres operaciones, tal y como ya ha hecho en otras ocasiones el Tribunal (SSTC 47/2004, FJ 5 y 80/2018, FJ 5). Primero, especificar el derecho sustantivo autonómico que presenta particularidades; segundo, señalar respecto de qué legislación procesal estatal se alegan las especialidades procesales autonómicas y tercero, averiguar si existe conexión directa 
justificable entre las peculiaridades del derecho sustantivo autonómico y las singularidades procesales de la norma impugnada.

Aplicando dicha doctrina, el TC llega a la conclusión de que la Ley ambiental vasca no puede considerarse una especialidad procesal autonómica. En primer lugar, porque el derecho sustantivo autonómico para este caso es de carácter medioambiental ( $\mathrm{y}$ no procesal). En segundo lugar, porque el precepto autonómico no introduce una especialidad, sino una ampliación, regulando la acción popular, que es competencia de derecho procesal general. Y, finalmente, porque esta ampliación no se conecta directamente con una peculiaridad del derecho sustantivo autonómico. Ni el Parlamento ni el Gobierno Vasco justifican la necesidad de esta singularidad procesal, como tampoco el preámbulo de la Ley ambiental vasca.

El Gobierno y el Parlamento Vasco, en sus escritos de defensa, sostenían además que la acción pública judicial prevista en el art. 3.4 de la ley ambiental vasca se justificaba por la necesidad de cumplir con los compromisos europeos e internacionales sobre justicia medioambiental, citando en concreto el Convenio de Aarhus. En respuesta a ello, el Tribunal considera que los tratados internacionales y el Derecho de la Unión Europea no sirven para enjuiciar la constitucionalidad de normas con rango legal. Asimismo, responde que la ejecución de los compromisos internacionales asumidos por España, sobre acceso a la justicia en materia de medio ambiente, debe hacerse conforme a las normas que establecen el orden de distribución de competencias entre el Estado y las comunidades autónomas. Niega pues, el Tribunal, que quepa esgrimir el Convenio de Aarhus ni el Derecho de la UE para aprobar una norma procesal en contravención del orden constitucional de distribución de competencias.

El Tribunal Constitucional concluye que solo el Estado, en virtud de su competencia general en legislación procesal, puede determinar los supuestos de legitimación para accionar en vía judicial. Añade, además, que la norma estatal de transposición del Convenio de Aarhus, esto es, la Ley 27/2006, de 18 de julio, por la que se regula el derecho a la información, participación y acceso a la justicia en materia de medio ambiente, ya aprueba en sus arts. 22 y 23 unas normas específicas que limitan la defensa del medio ambiente en vía judicial. El Tribunal Constitucional termina dando la razón al Tribunal Superior de Justicia 
del País Vasco, declarando la inconstitucionalidad del inciso del art. 3.4 de la ley vasca - aprobada curiosamente hace ya más de dos décadas - por invadir el ámbito competencial reservado al Estado en materia de legislación procesal. El Tribunal pone fin a la sentencia declarando que:

" (...) la extralimitación competencial apreciada es de origen y no deriva de que el Estado haya aprobado reglas específicas de legitimación procesal en materia medioambiental ni de la mayor o menor coincidencia de la norma autonómica con tales reglas, ya que, como hemos recordado recientemente, «un precepto autonómico será inconstitucional por invasión competencial siempre que regule cuestiones que le están vedadas, con independencia de la compatibilidad o incompatibilidad entre la regulación autonómica controvertida y la dictada por el Estado» [STC 65/2020, FJ 8 D) a)]."

Resulta especialmente interesante mencionar que el representante del Parlamento Vasco, en su escrito de defensa, había citado diversas leyes ambientales de otras comunidades autónomas que también reconocían la acción pública en vía judicial, sin que hasta el momento hubiera sido ninguna recurrida constitucionalmente. Al respecto, responde el TC que la falta de impugnación de disposiciones previas de contenido similar al objeto del proceso resulta irrelevante en el ejercicio de su jurisdicción. En cierto modo, la resolución de esta sentencia asienta un precedente, dejando vía libre para la impugnación de las no pocas leyes autonómicas que regulan la acción pública o popular en vía jurisdiccional en materia de medio ambiente. Este es el caso, por ejemplo, de las leyes ambientales de Castilla-La Mancha (Ley 4/2007, de 8 de marzo), la Comunidad Valenciana (Ley 6/2014, de 25 de julio), Ias Islas Baleares (Ley 12/2016, de 17 de agosto), Cataluña (Decreto legislativo 1/2009, de 21 de julio), Madrid (Ley 7/1990, de 28 de junio), Galicia (Ley 8/2002, de 18 de diciembre) y Canarias (Ley 1/1999, de 29 de enero).

Asimismo llama la atención que, habiendo tenido la ocasión para entrar a valorar la constitucionalidad de una de estas leyes, el TC no lo hiciera, como sucedió en la STC 109/2017, de 21 de septiembre (referida a la Ley del Parlamento Balear 12/2016). En cualquier caso, es posible que con esta nueva sentencia 15/2021 se haya abierto la caja de pandora. Si se comienzan a recurrir todas estas leyes y se aplica la base de la presente sentencia, la acción pública para la defensa de 
los intereses ambientales puede quedar ciertamente debilitada. La declaración de la nulidad de esta acción en vía jurisdiccional podría constituir, en última instancia, una traba de tipo procesal que impediría la implicación de la ciudadanía y la sociedad civil en la protección de intereses difusos y colectivos de tipo medioambiental. 